\title{
Terrorisme, politique et société dans « Dawa » de Julien Suaudeau
}

\author{
Ranya MOHAMED KAMAR \\ Maître de Conférences \\ DLLF Faculté des Lettres \\ Université d'Helwan
}

\section{Introduction}

Le terrorisme est défini comme "une violence, ou la menace d'une violence, calculée pour créer une atmosphère de peur et d'inquiétude. »". Il se définit également comme étant le recours à la violence dans un but politique. Il consiste à l'usage de la peur, par des minorités en vue de déstabiliser puis détruire un ordre «politique » existant. Le terrorisme n'est pas synonyme de « guérilla » même s'il y a des ressemblances dans les objectifs et dans les méthodes. La guérilla est une stratégie militaire qui se rapproche des principes de la guerre régulière. Au contraire, les terroristes, toujours clandestins, maintiennent leur caractère de petit groupe secret. ${ }^{\text {ii }}$.

Si le terrorisme est alimenté par la pauvreté pécuniaire et intellectuelle, il y a cependant des recherches selon lesquelles il serait difficile de réduire l'émergence ou l'existence du terrorisme à des causes économiques. En effet, d'autres dimensions, sociopolitiques et institutionnelles sont à prendre en considération ${ }^{\text {iii }}$.

Dans notre article, nous nous proposons d'étudier le terrorisme et sa relation avec la politique et la société et ce, à travers le roman Dawa de Julien Suaudeau. Notre choix du roman s'est fait après une longue recherche sur les œuvres littéraires ayant pour thème «le terrorisme ». Cependant, notre choix s'est fixé sur Dawa car d'une part ce roman vient d'être publié en 2014, il est donc récent, et d'autre part, nous n'avons pas trouvé de travaux d'analyse proprement dits sur le roman, ce qui nous a encouragée à vouloir découvrir cette œuvre que nous estimons d'une grande importance.

Dans les quelques lignes qui suivent, nous présenterons un bref résumé du roman :

Assan, professeur d'université d'origine algérienne, désire venger son frère Kader tué par la police française. Il pense qu'ainsi il pourra d'une part devenir le héros de la famille et d'autre part conquérir le cœur de Zohra, la veuve de Kader, devenue sa femme. Pendant plus de dix ans, Assan vivait avec Zohra sans qu'il ne puisse la toucher. Il a décidé un jour de faire exploser toutes les gares de Paris. Pour cette fin, il a pu recruter cinq jeunes parmi ses élèves les plus démunis. Son plan élaboré, il ne lui restait que l'exécution. La jeune femme, au courant de son plan, le supplie de renoncer à son attentat et lui avoue enfin sa volonté de vivre avec lui une vie normale avec leur fille Leila, fille de Kader qu'Assan a adoptée. Zohra lui explique également que cela n'avait aucun sens de venger Kader puisqu'en réalité ce n'était pas son demi-frère : sur son lit de mort, la mère d'Assan avait révélé à la jeune femme que celui-ci était né d'une 
aventure avec un inconnu. Face à ces révélations et surtout devant l'espoir d'une vie heureuse avec Zohra qu'il aime tant, Assan renonce à ses plans destructeurs. Mais il était sans doute trop tard ...

En fait, le roman Dawa incarne la colère de son auteur, Jean Suaudeau, fatigué par le fait de trouver la France toujours plus sinistrée. Suaudeau est né en 1975 à Evreux d'un père coopérant dans l'armée et d'une mère surveillante de lycée. Il voyage au gré des mutations parentales : Alger, Evreux de nouveau, puis Paris, où il va intégrer Henri IV en terminale, avant de s'inscrire à Sciences Po. C'est à travers un sport, la boxe qu'il découvre un univers différent : d'un côté les copains des salles de boxe, en grande partie, enfants d'immigrés qui désertent de temps en temps la banlieue pour les rings parisiens, de l'autre les amitiés BCBG sur les bancs d'école, enfants de bonne famille promis à un bel avenir. L'auteur navigue d'un côté et de l'autre de ces deux mondes, dans une France qui lui paraît de plus en plus divisée. Il travaille quelques années à l'ambassade de France à Bakou avant l'an 2000, il tombe amoureux de sa future femme et rentre à Paris pour se lancer dans la critique du cinéma puis dans le documentaire. Jean Suaudeau vit actuellement aux Etats-Unis où travaille sa femme.

Dawa est un roman «choc », ancré dans la réalité. Il est le fruit de huit ans de travail acharné pour mettre en lumière les zones d'ombre qui marquent la France. "Je suis exaspéré par ce contresens absolu qui nous fait croire à un clash ethnoculturel entre la France blanche et chrétienne et la France issue de l'immigration, alors que les constructions identitaires résultent d'une bonne vieille lutte des classes entre ceux qui sont dedans et ceux qui sont dehors $»^{i v}$, résume son auteur.

A travers Dawa de Julien Suaudeau, nous analyserons le thème du terrorisme comme étant un phénomène mondial, un fléau qui sévit dans le monde et ravage la planète jour après jour. Dans notre étude, nous adopterons l'approche sociocritique que nous estimons adéquate au thème choisi, et nous tenterons de répondre à la problématique suivante : le terroriste est-il victime ou bourreau ? Le contexte politique, économique et social peut-il créer un terroriste?

Nous commencerons par le milieu familial où a grandi le terroriste.

\section{1- Milieu familial}

Le milieu familial dans lequel a grandi le personnage du terroriste peut expliquer en grande partie les causes de sa dérive et révéler les facteurs importants qui l'ont poussé à opter pour la violence et le meurtre. Assan a-t-il les gènes de la violence comme son père et son frère ? A la question : «Naît-on violent ou le devient-on? », Thomas Heams, enseignant chercheur en biologie moléculaire à Agro Paris Tech, répond que «Les comportements violents ou criminels sont des phénomènes très complexes, infiniment sociaux qui ne peuvent pas être causés seulement par la génétique »".

\section{1-1 Le père :}

Assan Bakiri, ce professeur d'université, qui porte le nom de sa mère et dont la vie semble tranquille et banale, est en réalité le fils d'un des plus grands généraux du FLN : Al-Mansour. 
Son père avait participé à la guerre de libération de l'Algérie : "Dans les années cinquante, et jusqu'en 1962, Al-Mansour avait été l'une des grandes figures du combat pour l'indépendance (...), un général sans merci (...), et un terroriste dont les campagnes sanglantes restaient gravées dans la mémoire des Aurès. Sa bravoure et la cruauté que lui prêtait la geste indépendantiste avaient fait de lui un héros populaire (...). $\gg^{v i}$

Si Al-Mansour ainsi que d'autres combattants du FLN étaient considérés par la France comme des «terroristes », ils seraient et demeureraient pour leurs compatriotes des « résistants » qui s'étaient livrés à la tâche honorable de libérer leur pays. A l'époque, comme aujourd'hui, le mot « terroriste » met l'accent sur les méthodes employées, et a une forte connotation péjorative. Dès la première série d'attentats du FLN, le communiqué du gouvernement français évoque des actes « commis par de petits groupes de terroristes » tandis que les attentats sont qualifiés de «menées criminelles ». Plus tard, la loi sur « l'état d'urgence » précise qu'elle s'efforce de lutter contre des « terroristes ». Au contraire, bien sûr, ni le FLN ni l'OAS n'emploie ce lexique pour s'auto-désigner : ils sont des « résistants », des «combattants ». La guerre d'indépendance algérienne est déclenchée, dans la nuit du 31 octobre au 1er novembre 1954. ${ }^{\text {vii }}$ Selon Pierre Spiteri, professeur émérite des universités : «(...) en différents points du territoire algérien, mais plus particulièrement dans le sud-est constantinois et dans la région des Aurès, à la limite de la frontière tunisienne, une trentaine d'attentats sont revendiqués par le F.L.N : assassinats (...), attaques de bâtiments (...), sabotages (...). (...) Ces agressions visent principalement les symboles de la présence française en Algérie : édifices publics ainsi qu'exploitations agricoles et forestières européennes (...). »viii

Dans les premières pages de son livre, Julien Suaudeau nous peint une scène de sang et d'horreur où l'innocence d'un enfant et sa vie furent dévastées à jamais devant les corps de ses parents français égorgés par Al-Mansour et des membres du FLN.

«(...) le garçon pose la nuque de sa mère sur l'épaule de son père, puis se tourne vers l'homme en train d'essuyer son couteau.

- Si tu ne me tues pas maintenant, je jure que je te tuerai un jour.

- Tu n'as pas envie de vivre?

- Je vivrai pour te tuer, et j'aimerai le seul jour de ta mort. Al Mansour s'approche et lui colle la pointe de la lame encore humide sous la gorge.

- Tu as la vie devant toi pour y penser, fils. Souviens-toi de mon nom. $»^{i x}$

Cette famille, comme d'autres, a péri car elle représentait le colonisateur, l'ennemi qui s'était emparé du pays et avait réduit, pendant de longues années, la population indigène à vivre sous le joug d'une occupation oppressante et humiliante. Cependant, rien ne pourrait excuser ou justifier une telle violence.

Ce dialogue poignant entre un enfant et le tueur de ses parents ne peut qu'indigner le lecteur face à la cruauté de l'acte. Les mots «corps, émotion, froid, larmes » qui apparaissent dans la page 13 marquent le choc du garçonnet devenu orphelin en un instant par le crime d'Al-Mansour. 
Al Mansour, considéré par les uns comme terroriste et par d'autres comme héros, rêvait de libérer le Proche et le Moyen-Orient. Après la guerre «la mort dans l'âme, il avait fini par démissionner de l'armée ${ }^{x}$ et s'engagea comme mercenaire dans divers pays du monde arabomusulman. Cependant, "malgré les causes perdues -Libye, Egypte, Syrie- où l'avait précipité dans les années soixante-dix son amour du danger et une version très personnelle de la chasse au bonheur, (...), il ne s'était jamais dérobé devant sa responsabilité et ses devoirs paternels. ${ }^{x i}$ C'est au yeux d'Assan la chose pour laquelle il pardonnait «les péchés » de son père : sa polygamie et sa religiosité qu'il mélangeait aux dogmes de la libération nationale pour en fin de compte réaliser une gloire personnelle. Al-Mansour élevait ses fils comme de "petits guerriers », et c'est chez Kader que "la transmission avait naturellement infusé »xii. De son côté, Assan n'avait rien contre la France et les sermons de son père restaient presque sans effet sur lui. Cependant, c'est la mort cruelle de son frère qui a, sans doute, créé le déclic.

\section{2-2 Le Frère :}

Kader était né en 1967, "fruit d'une amourette qu'Al-Mansour avait eu avec une danseuse de Pigalle connue des amateurs sous le nom de Charlotte (...). Charlotte est partie un an plus tard, ne laissant rien d'autre que le bébé (...). »xiii Après la naissance d'Assan dix ans plus tard, AlMansour, malgré sa vie mouvementée, n'a jamais abandonné ses fils. Le temps qu'Assan a passé avec son frère aîné avant sa mort était suffisant «pour que se nouent entre eux de solides liens fraternels. » ${ }^{\text {iv }}$

Assan ne s'est jamais remis de la mort de Kader Al-Mansour le 30 mars 1996 lors d'un incendie à Mulhouse. Le personnage de kader n'apparaît qu'à travers les souvenirs d'Assan. Cependant, nous découvrons qu'il était à la tête d'une bande "de desperados armés par les islamistes algériens. ${ }^{x v} \mathrm{Ce}$ frère aîné avait choisi le chemin de son père. Il se maria avec une jeune Algérienne : Zohra Makhlouf. "Quand Kader n'était pas occupé à mettre des cocottes-minutes remplies de clous sous les sièges des RER, il voyait une fille algérienne de Belfort (...). La belle Zohra. ${ }^{x v i}$ A travers la description de Kader, le lecteur perd toute compassion avec ce personnage peint comme violent et même parfois sadique.

Après la mort de Kader, Assan fait de brillantes études et enseigne à l'université, épouse Zohra, et élève Leila qui n'a ni vu ni connu son père. Il héberge plus tard Al-Mansour, affaibli et terrassé par la maladie. Bien que cette famille semble mener une vie tranquille, elle reste et demeure dévastée par la mort de Kader, surtout qu'Assan n'a pas une vie normale avec Zohra. Ceci va sans doute déclencher la haine qui poussera ce dernier à préparer un attentat.

\section{2- Désir de vengeance ou mégalomanie}

\section{2-1 Vengeance :}

Assan n'a jamais été heureux de vivre en métropole mais n'a pas eu le courage de retourner en Algérie. Comme nous l'avons cité, des liens forts l'unissaient à son frère.

"A l'époque où son visage était placardé dans tous les journaux, exhibé à la télévision, la seule chose qu'Assan voulait savoir, inconscient des risques qu'il 
avait pris pour venir jusqu'à lui, c'est s'il était vraiment responsable des bains de sang à Paris l'été précédent, comme tout le monde le prétendait, y compris sa mère. Kader n'avait pas réagi comme s'il n'avait pas entendu la question. Avant de partir, il lui avait simplement fait jurer de prendre soin de Zohra si jamais il lui arrivait malheur. C'était très important qu'il jure. »xvii

En effet, la richesse et la complexité du lien fraternel résident précisément dans le fait qu'il offre une permanence et une stabilité familiale. La fratrie assure également pour les enfants une fonction de soutien et d'unité. Après la perte de son frère, Assan était pénétré par un sentiment de révolte et hanté par "un puissant appétit de vengeance, aiguisé par la tristesse et la colère. » ${ }^{\text {viii }}$. Seul un acte terroriste pouvait lui permettre d'assouvir sa haine et de solder ses comptes « contre les servitudes maléfiques du Golem qui a massacré son frère, et à la poursuite duquel son père a fini par perdre la raison. " ${ }^{x i x}$ Comme la majorité des terroristes qui ont frappé la France, Assan est issu d'une famille d'Afrique du Nord, notamment du Maghreb : Tunisie, Algérie et Maroc. Selon Claude Robert, la coïncidence ethnique ne tient d'ailleurs pas du hasard puisque ces trois pays proviennent d'anciennes colonies ou protectorat français. Il subsiste sans doute un ressentiment de l'ex-colonisateur français, une sorte d'ambiance amour/haine qui pousse au passage à l'acte lorsqu'un certain nombre de conditions sont réunies. ${ }^{\mathrm{xx}}$

Il voulait par son acte marquer les esprits, frapper l'imagination et rester dans les mémoires. Il aspirait à quelque chose de spectaculaire. Mais il s'était résolu à faire sauter toutes les gares de Paris. «(...) six attentats simultanés dans les six gares de Paris, perpétrés par six enfants de la République. ${ }^{x x i}$ Le nom de code qu'il a choisi est « Dawa », « Dawa al-Islamiya » ${ }^{\text {xxii }}$.

Les années qu'il a passées en France, sa réussite professionnelle, son ascension sociale n'ont pu le réconcilier avec ce pays. La haine qui ronge Assan reflète incontestablement des difficultés d'intégration, une difficulté de faire jeu égal avec la population du pays d'accueil, sur fond d'un possible sentiment de rejet ou de désamour de part et d'autre. Il s'agit sans doute d'un échec sur le plan de l'intégration. ${ }^{\text {xxiii }}$ Assan, comme beaucoup d'émigrés, n'a peut-être pas su trouver ses repères en France et n'a pas réussi à réaliser un équilibre entre la conversation de son identité et l'adaptation à une nouvelle société.

\section{2-2 Mégalomanie :}

Outre son désir de vengeance, Assan amoureux de Zohra, voulait lui prouver que son courage égalait ou surpassait celui de son frère Kader, considéré comme un héros au sein de la famille. Assan voulait conquérir le cœur de Zohra, même s'il serait mort. Il voulait devenir unique à ses yeux : une certaine mégalomanie sans doute. Selon certaines études, nous sommes tous mégalomanes, mais à divers degrés. Pour Denise Lachaud, psychanalyste, la mégalomanie devient pathologique lorsqu' "on constate un mépris radical de ce que peut penser ou éprouver le prochain. Et surtout lorsqu'on observe des désirs de destruction de l'autre ou de soimême. »xiv 
Assan a pris presque quatre ans pour mûrir son projet. Lorsqu'il était découragé, seule "l'avidité de regagner le cœur de Leila et de prouver à Zohra qu'il valait bien son mari, mort hérö̈quement au combat ${ }^{x x v}$ le poussait à persévérer et à poursuivre son plan. Il était avide de destruction, affamé de néant: "Six bombes et le septième jour (...) le diable pourrait chômer. ${ }^{x x v i}$ L'idée que son acte pouvait faire trembler de peur toute une nation l'excitait. Comme tout terroriste, Assan fonctionne avec une pulsion de mort ayant pour but de détruire l'autre psychologiquement ou physiquement. Il développe une forme de mégalomanie qui le rendrait intouchable : «Demain (...) à l'heure du "téléfoot» et du «jour du Seigneur», la France assise devant son poste apprendra le peu de temps qui lui reste à vivre dans son confort maussade de grande nation en déclin. » ${ }^{\mathrm{xxvii}}$

Ce résultat, cet ultime objectif est sans doute une réalité imaginée. En effet, le terroriste s'invente et invente l'autre. Il imagine que cet acte fera de lui un héros, un homme grand défendant une cause et vengeant les siens. Assan a décidé ainsi, ce sera " la sainteté et le martyr pour l'amour d'une femme qu'il ne reverra jamais " ${ }^{x x v i i i}$ puisque son acte qui donnera la mort à des centaines, achèvera également sa vie.

\section{3- Préparation des attentats}

\section{3-1 Recrutement des jeunes :}

Pour mettre son projet satanique en exécution, Assan procède au choix d'un groupe de jeunes, futurs auteurs des attentats. Pour six bombes, dont une qui sera à sa charge, Assan se doit de recruter cinq jeunes. Son travail comme enseignant à l'université, en plus de son logement dans une cité de banlieue - les 3000 -, et les cours d'arabe qu'il donne gratuitement lui permettent de côtoyer quotidiennement divers types de jeunes, et d'accéder clandestinement à leur dossier. Selon les études, les endoctrinés sont avant tout des jeunes. Ils ont entre 15 et 21 ans (63\%) et les $37 \%$ restant entre 21 et 28 ans. Elément important : 40\% d'entre eux ont connu un épisode dépressif dans leur vie. Les proies ont un profil fragile, « hypersensible » ${ }^{\text {xxix }}$ : elles sont donc très faciles à manipuler.

Nous remarquons que les mots «barbares », «barbarie ${ }^{\mathrm{xxx}}$ et $«$ racaille ${ }^{\mathrm{xxxi}}$ sont constamment employés dans le roman pour désigner les pauvres et les plus démunis. C'est ainsi que l'auteur souligne le regard méprisant que porte la «bourgeoisie » ${ }^{x x i i}$ à l'égard des moins favorisés de la France. Un appel à l'indignation du lecteur.

Assan choisit ses recrues avec minutie. Audrey est une jeune fille "pas forcément majeure », "française de souche (qui) a grandi dans les foyers de la DDASS avant d'être placée en famille d'accueil (...). Elle s'est récemment convertie à l'Islam. " ${ }^{\text {xxiii }}$ Ali, Driss, Bruno et Karim ont tous plus ou moins le profil d'Audrey. Ce sont des êtres désespérés, envahis par un sentiment d'injustice et révoltés contre un sort qu'ils n'ont pas choisi. Ils accepteront de tuer et de se tuer à cause d'un manque d'opportunités socio-économiques, à cause de la marginalisation, du chômage et d'une frustration grandissante. 
En effet, le chômage est l'arme destructrice du développement d'une nation. Il entraîne les jeunes à faire des activités illégales et illicites. Les jeunes finissent parfois par prêter allégeance aux terroristes non par ambition, ni par passion, mais pour l'argent et pour la vengeance. : pour l'argent, parce qu'ils ne sont pas seulement des chômeurs, mais aussi des pauvres qui vivent dans des conditions précaires, et pour la vengeance, simplement parce qu'ils sont marginalisés par leur société. Le désespoir, pour sa part, anéantit la jeunesse. Une jeunesse désespérée est comme un enfant, facile à tromper. Dans un dialogue entre Paoli, agent de police, et son subalterne au sein du commissariat, l'auteur nous dévoile cette réalité désolante :

«- (...) dans une cité comme les 3000, combien y-a-t-il de gamins perdus qui ne demandent qu'à faire brûler le monde où ils vivent?

-Où ils ne vivent pas vous voulez dire. (...) » xxxiv

Ce sont des paroles révélatrices, frappantes et d'une véracité incontestable. Ces jeunes ne vivent pas, ils tentent de survivre dans un monde qu'ils voient hostile et inhumain. Selon Anne Tursz, de nombreux travaux en psychologie ont montré que les jeunes ne peuvent grandir, s'épanouir et devenir des adultes heureux, socialisés et responsables que si leurs besoins physiques, affectifs et éducatifs sont pleinement satisfaits, dès leur naissance, par les personnes qui s'en occupent, très généralement leurs parents, dans un cadre stable, aimant et sécurisant. Par contre, les carences affectives sévères, qu'elles soient le fait de familles naturelles défaillantes ou de discontinuité dans les placements, sont souvent associés à la survenue ultérieure d'une délinquance. Le terrorisme apparaît donc, dans ce contexte, comme une étape de plus pour exprimer sa colère dans des sociétés qui n'ont pas réussi leur politique d'intégration. ${ }^{\mathrm{xxxv}}$ En effet, les cinq jeunes qu'Assan avait choisi «étaient déjà tombés (...) loin dans le chaos intime et la haine de ce monde marchand qui ne voulait pas d'eux (...) » ${ }^{x x v i}$

Les jeunes recrues d'Assan nous rappellent le personnage de Taher dans La Violence et la dérision d'Albert Cossery. Ce garçon d'une intelligence remarquable qui, sous le poids de l'oppression et de l'humiliation intègre les rangs des extrémistes et lance une bombe sur le cortège du gouverneur. Il finit «les poings enserrés dans les menotes » ${ }^{x x v i i}$. Le personnage du terroriste apparaît également dans L'immeuble Yacoubian xxxviiid'Alaa El Aswany. Marginalisé parce que pauvre, Taha perd l'espoir en un avenir meilleur et se voit hanté par la haine d'une société qu'il juge injuste et factice. Il perdra la vie lors d'un acte terroriste.

\section{3-2 Lavage de cerveau :}

Ces recrues bien choisies, la tâche d'Assan n'était que minime. Ces « gamins » feraient ce qu'il attendait d'eux sans demander le reste. Il n'avait qu'à leur peindre le paysage d'une nouvelle société rebelle, d'une guerre civile nourrie par les différences ethniques et religieuses pour attiser le feu de leur haine et de leur colère. Ces jeunes, qui œuvrent avec lui et qui seront les membres du groupe « Dawa islamiya » n'ont en réalité aucune culture théologique. Ils ont subi un long et profond lavage de cerveau de leur professeur Assan, pour qui Dieu n'existerait pas : 
"Dieu s'il existait, ce dont il doutait de plus en plus insidieusement malgré le socle religieux de son éducation et les consolations dont il remplissait désormais les esprits faibles de ses cinq disciples $»^{x x i x}$ il se devait afin d'achever son dessein, de convaincre les esprits faibles de ces cinq disciples qu'ils seront des martyrs, des héros, le noyau d'une révolution localisée contre une société islamophobe qui les cantonne dans un statut d'étranger, de sous-citoyen, comme jadis elle refoulait les fous dans ses prisons.

C'est ainsi qu'il persuadait ces jeunes de mourir en martyr en se délivrant du fardeau de l'injustice tout en accomplissant des actes héroïques et en servant une cause noble : "plus grande que soi ». ${ }^{\mathrm{xl}}$ «Les péchés des martyrs », leur disait-il « sont pardonnés dès la première goutte de sang versé.» ${ }^{x l i}$ Dieu bénissait leurs actes : ils iraient au paradis où seuls la gloire, le bonheur et l'éternité les attendent.

Les mots clés du mode opérationnel était « la discrétion » : pas de réseaux sociaux ni même de prière à la mosquée, et «l'étanchéité » : si l'on se fait arrêter, on ne dit rien, on n'affiche que l'indifférence et l'ignorance. Leur relation avec Assan Bakiri ne devrait être due qu'aux cours d'arabe qu'il donnait aux jeunes de la cité. Une vidéo fut ensuite filmée avec minutie et diffusée sur toutes les chaînes de la France. En effet, la puissance du terrorisme est entièrement construite sur les media, horrifier et provoquer la contagion de la terreur.

«La caméra est mise en place, le décor aussi, les pains de plastia bien en évidence sur la table à côté des détonateurs, et la bannière DAWA AL-ISLAMIYA déployée au mur en version originale. Lettres arabes, effet garanti à la télévision. » xlii

Il faut « détruire Paris », ${ }^{\text {xlii }}$ présentait le commando Dawa. C'est ainsi qu'Assan et les jeunes issus de l'immigration ou Français de souche, sont résolus «à dévorer le sein maternel de leurs mâchoires carnassières. " ${ }^{x l i v}$ Et il va sans dire qu'à la suite de la diffusion de la vidéo, en vingtquatre heures, il y a eu des dizaines de lynchage de Maghrébins dans toute la France. Des femmes voilées et des imams ont été agressés. En effet, dans divers pays du monde, l'amalgame persiste toujours entre musulman et islamiste. Dans l'article « Islam, islamisme, terrorisme : un amalgame inquiétant», Georges Leroux et Olga Hazam estiment qu'il est important "d'éclairer le phénomène général de la terreur et de la violence, à une époque où cette terreur est rapportée, souvent de manière non élucidée, à un fondement religieux. La place de l'islam et de l'islamisme est ici en fonction de leur importance dans les débats actuels, mais nous ne proposons d'aucune manière une équation entre terrorisme, islamisme et islam. Nous pensons au contraire que cette équation est grevée d'une intolérable confusion et nous croyons que plus nous laisserons cet amalgame envahir le champ politique de notre rapport à l'islam, plus nous verrons se creuser le fossé déjà largement ouvert entre les pays musulmans et les pays occidentaux. (...) Faire un pas de plus, comme on n'hésite plus à le faire si souvent en associant directement l'islamisme au recours à la terreur, c'est donner un tour de vis dangereux à une interprétation déjà fallacieuse du lien entre l'islam et l'islamisme. ${ }^{x l v}$ 


\section{4- Renoncement « tardif » aux attentats}

\section{4-1 Amour et espoir :}

Les bombes ont été fabriquées et la vidéo diffusée. Le plan a bien été élaboré, et malgré l'arrestation d'Audrey, puis sa libération, rien ne changera, tout se déroulera comme prévu et Soul remplacera la jeune fille. Les moindres détails avaient été calculés et pris en considération, une seule probabilité avait échappé à Assan : Zohra, pour qui il allait accomplir cet acte, Zohra, son épouse qu'il n'avait jamais touchée, l'aime et se déclare prête à vivre avec lui, ici et maintenant, une vie normale, entre mari et femme, une vie faite d'amour, une vie de famille avec Leila. C'est alors que toute la haine qu'Assan portait en lui disparaît, l'espoir renaît dans son cœur, dissipant tout désir de vengeance ou de gloire, ne laissant place qu'à l'amour de la vie.

«- Tu ne vas rien faire Assan. (...) Tu vas me promettre que tu ne vas rien faire, et la vie va recommencer comme avant, simple et heureuse.

- (...) Qui vengera Kader, si moi je ne le venge pas?

- Tu n’honoreras pas sa mémoire en sacrifiant ta vie à cette vengeance (...). La vie va recommencer comme avant, dit-elle en posant une main sur la sienne (...). Tous les trois, il faut qu'on tourne la page, maintenant. On a besoin de toi. "xlvi

Assan a découvert l'amour partagé : cet amour lui donne une énergie presque vitale qui le pousse désormais vers la vie. Il se sent réconcilié avec le monde. L'amour est sans doute, selon Pierre Teilhard de Chardin "la plus universelle, la plus formidable et la plus mystérieuse des énergies cosmiques. $»^{x l v i i}$

C'est ainsi qu'avec l'espoir, on reprend goût à la vie, c'est ainsi qu'avec l'amour, on désire rattraper le temps perdu. Il suffit de vouloir vivre et de vouloir être heureux : "Il veut vivre, vivre mille vies pour rattraper le temps perdu, et une seule, toujours la même, pour marcher à ses côtés dans Paris, lui faire l'amour, la regarder, parler avec elle, être toujours là où elle se trouve $»^{x l v i i i}:$ Zohra.

Subitement, Assan découvre que la vie est précieuse. Il a désormais un objectif et une famille. La mort qu'il allait se donner en se faisant exploser dans les attentats, a été substituée par un désir ardent de vivre. En effet, selon Freud, dans ses remarques sur l'opposition de la similarité de l'état amoureux et du suicide : quand on est amoureux, le moi est subjugué par un «bon objet » surinvesti, tandis que dans le suicide, il l'est par un «mauvais objet ». ${ }^{\text {xix }}$

"L'amour nous change et nous construit. Cette fonction supérieure permet au cerveau d'évoluer»l, souligne Francesco Bianchi Demicheli, docteur en médecine et spécialiste en psychiatrie et psychothérapie à l'Université de Genève.

Ayant renoncé à ses projets démoniaques, Assan se défoule dans une parole à Soul, quand le père de celui-ci fut emmené à l'hôpital : "Regarde comme la vie est précieuse » dit-il au jeune 
homme, qui fût surpris par les propos contradictoires de son professeur. Assan, avec ses longs discours sur la vie qui n'a aucune valeur, avec ses affirmations que dans des siècles et des siècles, les enfants apprendront leurs noms et se souviendront de lui et de ses camarades martyrs comme les libérateurs de l'Islam sur cette terre d'oppression, ce même Assan vantait la préciosité de la vie. Soul n'en revenait pas ! Il s'est senti trahi : "Ce n'est pas ce que vous disiez cet après-midi », lui lance-t-il, «Et ce n'est pas ce qui est écrit dans le Coran. " ${ }^{\text {li }}$ Le jeune ne voit plus Assan du même œil. D'ailleurs, il s'est toujours douté sur les intentions de celui-ci, car pourquoi un agrégé d'arabe et professeur d'université, auteur d'articles dans des revues prestigieuses et indépendant financièrement "voudrait-il à ce point se venger de la France qui lui a tout donné et chercher à se tuer? Quelle calamité secrète peut être assez immense pour gâter le miel de ces bénédictions?» lii

Si Assan avait renoncé aux attentats, et qu'il voulait plus que jamais vivre et vivre heureux, comment pourrait-il dissuader ces jeunes?

«(...) après leur avoir injecté le venin d'Al-Mansour, après les avoir dressés des mois et des mois dans le culte de représailles légitimes, cultivé leur psychose identitaire jusqu'à ce qu'elle devienne une réalité objective, la seule vision du monde qui explique à leurs yeux ignorants l'étendue de leur misère, comment (...) a-t-il pu espérer qu'il saurait les en faire sortir, simplement comme on se réveille d'un mauvais rêve? "liii

Le retour de Zohra a éveillé en lui toutes les émotions qu'il croyait disparues en suivant le chemin de son père. Maintenant, tout est fini, il renie cette pulsion de mort qu'il sentait dans ses veines. Maintenant "il a envie de croire qu'il est l'homme le plus heureux de France." liv Cependant, Jeau Suaudeau qui nous a si bien décrit le personnage d'Assan avec ses diverses facettes contradictoires et parfois incompréhensibles, nous prépare à un dénouement qui transmettra son message : jamais la manipulation et la violence ne seraient et ne pourraient rendre justice.

\section{4-2 Intervention policière :}

Daniel Paoli est commissaire dans la police antiterroriste, au Ministère de l'Intérieur, il y a bien longtemps. Agent chevronné et expérimenté, il savait détecter et traquer les terroristes les plus dangereux et les plus imprévisibles. Ce n'est pas par hasard que Paoli a choisi de faire son entrée dans la police. Pour lui, ce travail n'était pas un simple gagnepain : "c'était une quête primitive, sa réponse d'adulte à la promesse qu'il s'était faite en se réveillant au dispensaire de Batma. Ce matin-là, au lendemain de l'exécution de ses parents. » $1 \mathrm{v}$

Paoli est bel et bien l'enfant français qui a vu Al-Mansour assassiner et égorger ses parents devant ses yeux en Algérie pendant la guerre de libération. Sa vengeance n'allait pas commencer aujourd'hui en découvrant qu'Assan était le fils d'Al-Mansour, elle avait commencé depuis des années. L'incendie où Kader avait trouvé la mort n'était que 
l'œuvre de Paoli. C'est lui qui avait mis le feu à une fourgonnette bourrée de jerricans d'essence qu'il avait lui-même garée sous la planque de Kader et ses copains. Il avait ainsi fait éclater le cœur d'Al-Mansour : "la chair de sa chair avait péri de la façon la plus épouvantable qu'on puisse concevoir et c'était là une avance conséquente sur le prix de sa vengeance. »lvi

Il allait maintenant tuer son second fils Assan, et sa petite-fille Leila, dont il avait découvert l'existence. Il ne se laisserait pas devancer par son propre service, pour Paoli "la justice de ce pays n'a ni à juger ni à sauver le meurtrier de ses parents et sa descendance bâtarde. "lvii Habité par une vengeance et une haine que rien ne peut atténuer, Paoli, au courant des plans d'Assan, pénétre chez lui, tue Zohra qui s'interpose, et rate Assan qui s'enfuie avec une balle à l'épaule. La jeune Leila disparût aussi. Se trouvant nez à nez avec le vieil homme, Al-Mansour, Paoli s'apprête à lui faire payer toutes ses atrocités. Soudain, il découvre une silhouette, amaigrie, rongée par la maladie, le reste d'un homme qui ne sait même plus qui il est. Paoli avait attendu ce moment pendant des années, il avait sacrifié sa vie et celle des siens - son fils lui reprochait de ne lui avoir jamais accordé d'attention, ni d'avoir passé assez de temps avec lui - en attente de ce moment qui n'a plus de sens. Lui, qui a vu son père crever "comme une bête de ferme » ${ }^{\text {viii }}$ quand il avait onze ans, qui a choisi pour le venger "d'abattre la lignée des Arrache, et d'achever le patriarche avec un couteau de chasse, comme il a tué son père $»^{l i x}$, voit devant lui un squelette qui ne se rappelle rien. Anéanti, car sa vengeance ne servira plus à rien, il laisse le couteau et sort de la maison.

Après toutes ces années d'attente, cette traque du tueur de ses parents, les sacrifices qu'il a dû faire pour arriver à ce jour, tout n'a servi à rien. Al-Mansour, atteint d'alzheimer et affaibli par la vieillesse, n'est plus qu'un mort vivant. Devant l'absurdité de la situation, Paoli retrouve cette lucidité qui suit parfois le choc, et prononce ces mots, ou plutôt cette leçon de vie : «(...) Ne laisse jamais la haine prendre le contrôle de ta vie, et prends soin de ta famille. Adieu fils », ${ }^{\mathrm{lx}}$ dit-il à Franck.

\section{4-3 Rôle de la CIA et du Qatar :}

Tous les préparatifs pour les attentats terroristes qu'Assan voulait perpétrer, depuis le recrutement des jeunes jusqu'à la vidéo diffusée, étaient suivis en détails par les services de renseignements américains. En effet, "Alan G. Pearson, chef du bureau parisien de la CIA, vétéran des forces spécialisés » ${ }^{l x i}$ avait ses hommes partout dans Paris. Les moindres faits et gestes d'Assan, comme d'autres, lui étaient envoyés dans des rapports secrets.

"(...) il a commencé sa carrière dans le nord de l'Iran, dans les années quatrevingt, au milieu de la guerre avec Bagdad. A la connaissance de Paoli, c'est l'un des seuls hauts responsables du renseignement américain à parler l'arabe et le farsi. »xii 
Quand Sélim Arif, musulman chiite et informateur de Paoli, a voulu, par solidarité avec Assan, le prévenir qu'il avait été détecté par la police antiterroriste : «Tu es sur le radar de l'antiterrorisme »lxiii, il fut achevé d'une balle aussitôt descendu de la voiture de ce dernier. Cette balle, dont les policiers français ignoraient la provenance, et ce crime dont ils n'ont pas pu identifier l'auteur, n'était que l'œuvre de la CIA. Pour Sélim, Assan valait mieux que son père et son frère, il n'était pas un assassin : (...) tu ne tueras que des innocents et tu auras une mort absurde, parce que ceux que tu entendais punir te survivront. On ne tue pas un pays, Assan (...). » ${ }^{\text {lxiv }}$

Après sa rencontre avec Zohra, quand Assan a réalisé qu'il pouvait être heureux avec la femme de sa vie, lorsqu'il a décidé de vivre et d'abandonner son projet terroriste, c'est Pearson qu'il a trouvé devant lui, froid, indifférent, presqu'inhumain, lui ordonnant de poursuivre son plan comme prévu jusqu'au bout : Paris devait exploser !

«Nous ferons de notre mieux pour que personne ne vous empêche de parvenir à vos fins. Personne, y compris les services français. " ${ }^{l x v}$ Les quatre ans de préparation, les quatre ans de sacrifices pour rendre justice à Kader et «accomplir sa part de la revanche familiale contre la France » "xvi n'allaient que servir la CIA. Assan est dégoûté : "cette pensée lui donne envie de vomir. "lxvii Il se rend compte de l'absurdité de la situation. Finalement, nous ne sommes tous que des pions entre les mains de grandes puissances.

«- Et si je refuse?

- Vous serez livré aux services français, et votre père ira passer le peu de temps qui lui reste à vivre dans une prison fantôme que nous avons établie depuis des dizaines d'années chez nos amis est-européens. » 1xviii

Suaudeau nous révèle une des facettes de la politique américaine, l'ingérence secrète dans les affaires des pays, les ficelles que celle-ci tire de loin pour tout dominer et tout orchestrer afin de servir ses intérêts et garantir son hégémonie sur le monde.

"Quels que soient le résultat du grand barnum électoral mis en scène tous les quatre ans et la bonne foi du vainqueur quand il prête serment, le vrai pouvoir de Washington demeure une machine tentaculaire, comme dans ses fins, manouvrée par des intérêts privés dont la cupidité et la volonté de puissance ne connaissent aucune limite. Cette pieuvre (Les Etats-Unis) veut tout entendre, tout voir et tout savoir (...)» ${ }^{\text {xix }}$

Une analyse détaillée et lucide faite par Paoli qui reflète une certaine vision des dessous de la politique américaine.

Outre les Etats-Unis, le Qatar est l'un des acteurs politiques secrets sur la scène internationale. Dans son livre, Julien Suaudeau évoque le rôle suspicieux du Qatar. Depuis plusieurs années, ce richissime état du Golfe s'est lancé dans une politique d'investissement dans le monde, et plus particulièrement en Europe. En dépit de son rôle controversé dans le soutien de plusieurs courants islamistes, le Qatar est bien implanté dans le paysage économique et diplomatique de 
la France. ${ }^{\text {lxx }}$ La répétition du mot «Qatar» et de ses dérivés «Qatari » et «Qatariser » ainsi que le mot «Doha » que l'on voit notamment dans les pages : 20, 22, 25, 32, 33, 50, 70, 75, 76 et 111 marquent l'omniprésence de cet Etat pétrolier en France et transmet au lecteur le danger que cela représente. En effet, A travers les 642 pages qui constituent le roman de Suaudeau, le Qatar occupe une place qui n'est pas la moindre. L'implantation des réseaux qataris, les investissements énormes du Qatar en France sont accompagnés d'une présence secrète dans des milieux et lieux religieux musulmans ainsi que chez des jeunes, notamment pauvres, de ce culte. En effet, Larbi Ferhaoui, le fameux imam qatari, supposé représenter l'Islam modéré par une apparence aussi spirituelle qu'inoffensive contraire aux «spectres sataniques, hâves et barbus » ${ }^{l x i}$ d'Al Qaïda , d'Aqmi, n'est en réalité que "le poisson pilote du Qatar dans son entreprise de coloniser l'économie, la société et la politique française, du Royal Monceau et des hôtels particuliers de l'Ile Saint-Louis au 93, en passant par les doudous présidentiels du $P S G$ et les antichambres de la République » ${ }^{l x x i i}$. C'est une nouvelle politique qui s'instaure dans le monde, nous ne sommes plus à l'époque de la guerre des armes, mais selon l'auteur à celle de "soumettre l'ennemi sans combat. » "xxiii Ferhaoui, entre autres, avait donc pour mission d'endormir la vigilance du maître des lieux, jusqu'à ce que les clés tombent de sa main et que la porte s'ouvre en grand : politique que le Qatar entreprenait non seulement en France, mais également au Royaume-Uni et dans d'autres pays et régions du monde. Cette stratégie, à très long terme, vise selon l'analyse de l'auteur à servir les intérêts des Qataris et d'anticiper «sur l'épuisement de leurs réserves de gaz. Une stratégie de substitution pour assurer leur avenir. "lxxiv

Selon l'opinion de Salem Benammar, docteur en science politique et auteur de plusieurs ouvrages, la participation du Qatar dans les fleurons du capitalisme français ne doit en aucune manière être perçue comme étant de simples investissements ou des placements pour garantir le futur du pays. Il en est de même pour son rachat du club de football le PSG, et l'acquisition des droits de télévision du championnat de France de football. Le Qatar œuvre en vue de se positionner comme un acteur incontournable du capitalisme et du football français. Il tente de dominer les secteurs-clés de la société française afin de pouvoir peser de tout son poids financier et médiatique sur les orientations politiques de la France et notamment dans le cadre du processus de l'islamisation rampante de la société française qui devient de plus en plus perceptible. Par conséquent, il ne s'agit aucunement d'un partenariat capitalistique ou humanitaire, il s'agit surtout d'une intrusion impériale du Qatar dans les zones urbaines et dans le monde des affaires ainsi que dans le sponsoring sportif. ${ }^{\text {lxxv }}$

C'est la volonté de puissance et de richesse qui régit tout, sous une apparence élégante de relations amicales, de coopération commune et de projets pour le développement et la stabilité. Les complots sont bien dissimulés par des attentions de bienfaisance : "La société française doit être conquise en douceur, pas par la confrontation. L'action violente, c'est une ruine du siècle dernier. " ${ }^{l x x v i}$ 


\section{5- Cercle vicieux}

Tout est un cercle vicieux, fermé, fait de complots, mais aussi d'oppression, d'humiliation, de haine, et par conséquent de violence.

\section{5-1 Explosion :}

Après la mort de Zohra et la perte de Leila, Assan, blessé et attendant la mort, disparaît. C'est avec son sac à dos contenant une bombe qu'on le retrouve à nouveau. L'auteur nous le montre, contemplant le paysage calme et paisible de familles et d'enfants devant une école primaire, il contemple un paysage dont il ne fera jamais partie et qui ne sera jamais le sien. Le lecteur s'attendait sans doute à ce qu'il succombe à ses blessures et décède, loin des gares de Paris qu'il voulait faire «sauter ». Cependant, c'est un coup de théâtre, et c'est l'explosion fatale qui vient dissiper le calme de cette scène. La haine a encore pris le pas et la vengeance a tout ravagé. En commettant cet acte, Assan n'est plus l'être humain, c'est le monstre que le désespoir a généré. Des parcelles de morceaux humains s'envolent pour atterrir partout, un tremblement fait vibrer Paris, et là, devant l'école, les cadavres et les blessés sont partout...

Le mal qu'Assan a ressenti, il a voulu le transmettre à toute une société. Tous devaient payer pour la perte de Zohra, de Leila et de sa vie ... Ils devaient payer de ce qu'ils ont de plus cher. Assan s'est senti victime, mais être victime justifierait-il de tuer? La vengeance, le meurtre et la terreur des innocents, des êtres purs, des enfants, sont-ils les moyens d'obtenir justice?

\section{5-2 Suicide et arrestation :}

Quant aux jeunes recrues, à peine sorties de l'adolescence, elles ont été arrêtées à l'exception de Bruno. «Baguette », comme on le surnommait, désespéré, fatigué d'être traqué par la police, et craignant d'être arrêté, emprisonné, s'est suicidé sous les rails d'un train. Ses amis ont tous reçu la photo du suicide sur leur téléphone. Bruno est victime : victime de sa famille, de la police, d'Assan ou de toute une société ?

"(...) la victime, de sexe masculin, n'a pas encore été identifiée, son pronostic vital serait engagé. Des rumeurs circulent déjà selon lesquelles ce garçon aurait sauté sur la voie pour tenter d'échapper à un contrôle de police, et le téléphone arabe du 93 est saturé par des appels à l'émeute.

- Les bâtards », dit Karim entre ses dents. 1xxvii

La marginalisation a mené au désespoir, le désespoir à la vengeance, la vengeance à l'oppression et ainsi de suite. C'est un cercle vicieux entre les jeunes des quartiers défavorisés, stigmatisés et les autorités, la police : la police qui avait traqué Momo, qui l'avait arrêté pour l'obliger à devenir informateur ou à être arrêté pour détention de stupéfiants, alors que le jeune menait une vie pauvre mais calme où il n'y avait que sa mère, sa copine et la boxe. L'auteur 
dénonce une injustice destructrice. Nous sommes face à des actions et réactions insensées, absurdes où s'affrontent et se confrontent victimes et bourreaux.

Selon une étude de Chloé Guille, publiée par le Centre International pour la Paix et les Droits de l'Homme : "Les politiques actuelles de certains pays qui tendent à vouloir construire une hyper-sécurité se basent sur une réflexion homogénéisante du processus de radicalisation et se font au détriment des droits fondamentaux, pouvant alors engendrer une marginalisation de certains individus qui par un effet de cercle vicieux, pourraient tendre à s'intéresser à ce que les gouvernements veulent combattre. ${ }^{\text {lxxviii }}$ Le résultat est désastreux comme le montre Julien Suaudeau dans Dawa.

\section{5-3 Malaise d'une société :}

Assan a mené une guerre qui n'est pas la sienne, puisqu'Al-Mansour n'était pas son vrai père et donc Kader n'était pas son demi-frère. Paoli aussi a perdu sa vie en traquant le tueur de ses parents et "il aurait voulu ne pas être à l'image de son ennemi, prisonnier de sa vengeance, laisser la justice faire son cuvre, la mémoire sereine, libérée enfin de la malédiction et de sa sale guerre. "lxxix Pour leur part, les jeunes ont été manipulés et entraînés dans un combat qui ne faisait que concrétiser la volonté d'Assan en exploitant leur rage et leur désillusion. Mais alors, la France, que fait la France?

Julien Suaudeau ne lésine pas dans la critique de la politique française et des autorités à l'égard des quartiers difficiles et notamment des étrangers, issus de l'émigration.

«Il pleut toujours où c'est mouillé, aux 3000 et sur les autres terrains vagues de la République. Ça fait des années qu'il n'y a plus rien, plus de service public, digne de ce nom, ni police, ni école, ni hôpital, ni transport en commun, et quand l'Etat réinvestit les lieux, c'est avec l'uniforme et la violence d'une armée d'occupation - des pauvres qui tapent sur des pauvres. "lxxx

La police traque les jeunes. La force et parfois la violence sont les moyens déployés pour soumettre les cités comme les 3000. Mais est-ce le moyen efficace ? Est-ce le moyen pour combattre « la barbarie » ou au contraire de la créer et de la rendre plus violente que jamais ?

Bertrand Badie, professeur des universités à l'Institut d'études politiques de Paris affirme : "Mettre l'accent sur la dimension répressive est une façon dangereuse de nier ou de simplifier les causes profondes de ces violences. Devant une situation si tragique, tout gouvernement doit d'abord parer au plus pressé pour protéger ses citoyens, et nous disposons de l'arsenal juridique nécessaire. Mais il doit ensuite concentrer ses efforts sur des politiques publiques ambitieuses, qui s'attaquent aux racines du mal et privent les entrepreneurs de violence d'une clientèle qui leur sert d'agents. Procéder autrement, en recourant à des moyens qui démantèleraient l'État de droit, ne ferait qu'installer un climat de guerre que recherchent de tels entrepreneurs et dont la France doit se préserver. » Quant à Yasmine Boudjenah, elle 
estime que les actions de l'Etat doivent cibler les commanditaires d'une part et le terreau d'une autre part. Selon elle, les commanditaires seraient l'Arabie Saoudite et le Qatar qui soutiennent financièrement les groupes islamistes et le terreau est la France : "Le terreau, lui, il est bel et bien dans notre pays. Il a pour noms: chômage, échec scolaire, inégalités territoriales, discriminations, stigmatisations, pseudo-identité nationale construite sur le rejet d'une partie des citoyens, mais aussi la négation de pans entiers de l'histoire de notre pays. » Pour sa part, Paul Quilès, ancien ministre, précise que les Français ont manifesté à la fois un ferme refus de l'obscurantisme et de l'islamo-fascisme, mais qu'ils aspiraient également à une France plus solidaire, où les différences sont gommées. ${ }^{\text {lxxxi }}$

En effet, dans son livre, Julien Suaudeau dénonce la régression des libertés et l'acharnement des forces de l'ordre contre les jeunes des quartiers pauvres.

«La police est là pour tenir les barbares à distance de la grande ville civilisée, pour les contrôler aux facies dix fois par jour quand ils ont le malheur de s'y aventurer-certainement pas pour faire cesser l'état de barbarie. "lxxii

Pour sauver la France, l'auteur propose le rejet de la stigmatisation, et du règne de la bourgeoisie de qui les pauvres sont contraints de rester à distance: loin des beaux quartiers, des universités prestigieuses et des postes à pourvoir. "Rien ne sauvera la France tant que l'on vivra sous le règne des identités fabriquées, stigmatisation d'un côté et autosuggestion de l'autre, entre l'Arabe, le Basamé, le Musulman, l'Etranger, le fauteur de troubles et l'envahisseur. " ${ }^{\text {lxxiii }}$ Des paroles qui interpellent et invitent à une longue réflexion.

Peut-être la politique française vise-t-elle ainsi à défendre «l'identité nationale » face à un danger qui lui serait fatal. Toutefois, selon une étude publiée par l'ONU, cette perception de danger provient, entre autres, de publications d'articles sur la supériorité de la civilisation occidentale et plus précisément de l'émergence de la nouvelle idéologie du « conflit des civilisations et des religions » entre l'Occident et le reste du monde, tantôt réduit à une religion, l'islam, tantôt identifié comme « allogène » par l'ethnie et la culture. Ses nouveaux théoriciens, dans le monde académique et médiatique, recyclent ainsi la rhétorique manichéenne de la guerre froide dont ils sont souvent originaires, entre la civilisation et la barbarie, la modernité et l'obscurantisme, les droits de l'homme et les dictatures. ${ }^{\text {lxxiv }}$

Freud affirmait déjà, en réponse à l'angoisse civilisationnelle des élites face aux hécatombes de la Première Guerre mondiale : "La question n'est pas que nous soyons tombés si bas (en nous entretuant) mais la question est que nous n'étions pas aussi élevés que nous le pensions $»{ }^{1 \times x x v}$ Sans doute, avait-il raison ... 


\section{Conclusion}

Dawa est un roman choc qui dissèque aussi bien le monde de la police, les dessous de la diplomatie que les raisons du terrorisme et ses conséquences désastreuses. Julien Suaudeau appelle à une vision plus humaine, à une compréhension plus profonde et à des solutions plus adaptées aux problèmes et aux difficultés des jeunes marginalisés, déprimés parce que défavorisés par le sort.

Accepter l'autre avec toute sa différence et lui accorder sa place dans la société est la seule et unique solution. Racisme, marginalisation, stigmatisation et humiliation ne mèneront qu'à une guerre civile. "Le grand malaise, la guerre civile qui vient, cette guerre que nulle ne saura arrêter parce qu'elle est sans autre motif que l'aberrante folie de l'identité, nous sommes la France et vous êtes des barbares, non c'est nous qui sommes chez nous maintenant et vous allez payer pour vos malheurs. ${ }^{l x x x v i}$ Tel est le scénario que Jean Suaudeau invite à éviter.

Diverses recherches ont été faites à ce sujet afin d'aider les décideurs à orienter leurs décisions et leurs politiques vers les aspirations des jeunes. Dans une étude publiée par l'Institut malien de recherche action pour la paix, les chercheurs ont recommandé aux Etats d'appuyer les initiatives pour les jeunes en impliquant toutes les parties concernées; d'assurer un accompagnement communautaire à l'insertion des jeunes violents; de valoriser les groupes en rupture avec la société et de prendre en considération l'existence d'une professionnalisation de la violence au-delà des frontières nationales; d'assurer une continuité éducative entre familles $^{\text {lxxxvii }}$ et pour les musulmans, d'engager toutes les parties prenantes dans une meilleure compréhension de l'enseignement coranique pour valoriser les bonnes pratiques.

D'autre part, il est également indispensable dans ce contexte, de procéder à un programme de développement économique et social. Ce programme sera un instrument efficace contre la demande et l'offre du terrorisme : il permettra d'élever le niveau de revenu et l'expansion des classes démunies et des classes moyennes, ce qui réduira l'espace de soutien aux groupes terroristes. En outre, il est primordial de lutter contre les Etats sponsors qui financent le terrorisme.

Aux côtés des efforts devant être déployés au niveau des gouvernements et des responsables politiques, un autre rôle doit être joué sur le plan humain. C'est avec la compréhension, l'altruisme et l'amour que l'on peut vaincre le terrorisme. Si Dawa est un roman « cruel » par ses évènements, il ne faut pas oublier que l'amour et l'amitié y tiennent une place primordiale. Soul n'a pas fait exploser sa bombe de peur pour Momo, son ami, son frère, avec qui il a partagé des moments difficiles, avec qui il a traversé des épreuves pénibles, son confident, la seule personne sur qui il pouvait compter. L'amitié a donc vaincu la violence. La solidarité entre les minorités apparaît également dans le roman. Assan, n'a-t-il pas demandé à sa voisine palestinienne d'éviter les grandes gares de Paris ? «Bonne nuit Hayet. Gardez-vous des grandes gares dans les semaines qui viennent. ${ }^{l x x x v i i i}$ Sélim Arif n'a-t-il pas tenté de dissuader Assan 
avant qu'il ne s'enlise davantage dans son projet terroriste ? Même le renoncement d'Assan à sa vengeance était par amour à Zohra.

Cependant, à travers le roman, nous constatons que la haine est un choix, un choix dont l'être doit avoir la force de se défaire avant qu'il ne soit trop tard. «(...) chacun est responsable de sa haine ${ }^{l x x x i x}$ pensait Paoli. Comme chacun est responsable de son choix et que les circonstances ne justifient pas la violence, l'Etat également doit assumer ses responsabilités et prendre en considération, comme nous l'avons cité, les besoins des enfants et des jeunes, notamment les plus fragiles et les plus démunis.

$\mathrm{Si}$ «le défi du terrorisme et de la barbarie nous interpelle, il n'est pas nouveau. Voilà des siècles que l'humanité est en recherche de la civilisation, c'est-à-dire d'une civilisation de l'amour $»^{x c}$, affirme Jacques Garello, économiste et professeur émérite à 1'Université Aix Marseille. Le défi d'une société désarticulée nous atteint partout. Malgré cette réalité, il est important de croire fermement que même au milieu des obus qui éclatent et des canons qui crachent, il reste toujours l'espoir d'un matin radieux où l'amour de l'homme reprendra la place sur la cruauté d'une poignée d'individus. La seule arme efficace est celle décrite par Martin Luther King :

"L'obscurité ne peut pas chasser l'obscurité, seule la lumière le peut. La haine ne peut pas chasser la haine, seul l'amour le peut (...). Nous devons apprendre à vivre ensemble comme des frères, sinon nous allons mourir tous ensemble comme des idiots. $»^{x c i}$

\section{Notes}

${ }^{i}$ Deffarges Thierry, «Sur la nature et les causes du terrorisme », Une revue de la littérature économique ; https://www.cairn.info/revue-tiers-monde-2003-2-page-369.htm

${ }^{i i}$ El Khadir Mahmoud, «Le terrorisme, les causes et les remèdes », Université Mohammed I - Faculté de Droit - Licence 2005, https://www.memoireonline.com/01/07/330/m_le-terrorisme-les-causes-etles-remedes.html

iii Deffarges ,op.cit. p.378.

iv Lépine Élise, «Dawa, roman d'un Français en colère, Quand la fiction puise dans la réalité sociale, la littérature fait l'effet d'un coup de poing. La preuve avec le livre de Julien Suaudeau », http://www.lepoint.fr/culture/dawa-roman-d-un-francais-en-colere-30-03-2014-1807110_3.php

${ }^{v}$ Galanopoulo Léa, «Le gène de la violence : un instrument politique », https://sciences-critiques.fr/legene-de-la-violence-un-instrument-politique/

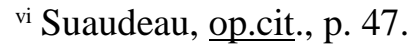

vii «FLN et OAS : deux terrorismes en guerre d'Algérie », In Revue Européenne d'Histoire / European

Review of History, vol. 14, n³, septembre 2007, p.325-342, https://halshs.archivesouvertes.fr/file/index/docid/541818/.../FLN_et_OAS.doc

viii Spiteri Pierre, Professeur Emérite des Universités, «La stratégie terroriste menée par le F.L.N : une stratégie hélas, efficace», http://www.clan-r.org/portail/IMG/pdf/La-strategie-terroriste-menee-par-leFLN-une-strategie-helas-efficace.pdf

${ }^{\text {ix }}$ Suaudeau, op.cit., pp.16-17.

${ }^{\mathrm{x}}$ Ibid., p.48.

${ }^{x i}$ Idem. 
xii $\underline{\text { Ibid., }}$ p.49.

xiii Ibid, p.48.

xiv Idem.

${ }^{\mathrm{xv}}$ Ibid., p.86.

xvi Ibid., p.90.

xvii Ibid., p. 150 .

xviii Suaudeau, op.cit., p.49.

xix Ibid., p.204.

${ }^{\mathrm{xx}}$ Robert Claude, «Terrorisme islamiste : quelles sont les vraies causes? »,

https://www.contrepoints.org/2017/01/02/261277-vraies-causes-du-terrorisme-islamiste

${ }^{\text {xxi }}$ Suaudeau, op.cit., p.50.

xxii Ibid., p.51.

xxiii Robert Claude, op.cit.

xxiv Taubes Isabelle, «I'm just a mégalo », www.psychologie.com/Moi/se-

connaitre/comportement/articles-dossiers/1-m-just-a-megalo

${ }^{\mathrm{xxv}}$ Suaudeau, op.cit., p.52.

xxvi Idem.

xxvii Ibid., p.102.

xxviii Ibid., p.204.

xxix 6Medias, «Terrorisme : quel est le profil des djihadistes ?», http://www.lepoint.fr/terrorisme-

quel-est-le-profil-des-djihadistes-php 19-08-2017-215001_23)

${ }^{\mathrm{xxx}}$ Suaudeau, op.cit., p.120.

xxxi Ibid., p. 120 .

xxxii Idem.

xxxiii Ibid., p.35.

xxxiv Ibid., p.37.

${ }_{\mathrm{xxxv}}$ Tursz Anne, «Pourquoi et comment devient-on terroriste ? , http://www.huffingtonpost.fr/annetursz/trajectoire-terroristes-france_b_7068514.html

xxxvi Suaudeau, op.cit., p.54.

xxxvii Cossery Albert, Euvres Complètes II, Editions Joëlle Losfeld, Paris, 2005, p. 337.

xxxviii El Aswany Alaa, L'Immeuble Yacoubian, Actes Sud, 2006.

xxxix Ibid., p.53.

${ }^{x l}$ Ibid., p.55.

${ }^{\text {xli }} \underline{\text { Ibid, }}$ p.337.

xlii Ibid., p.97.

xliii Ibid., p.176.

xliv Ibid., p.50.

xlv Leroux Georges, Hazam Olga, «Islam, terrorisme, un amalgame inquiétant »,

http://www.spiralemagazine.com/dossier-magazine/islam-islamisme-terrorisme-un-amalgame-

inquietant

xlvi Suaudeau, op.cit., p.p.422, 423.

xlvii Telhard de Chardin Pierre, paléontologue et philosophe «L'amour est la plus universelle, la plus formidable et la plus mystérieuse des énergies cosmiques » in «Qu'est-ce que l'amour ? Valeur, énergie ou sentiment?», http://www.etreproactif.com/quest-ce-que-lamour-valeur-energie-ousentiment-etre-ou-faire/

xlviii Suaudeau, p.473.

xlix Deffarges Thierry, op.cit., p.378.

'Telhard de Chardin, op.cit.

${ }^{\text {li }}$ Suaudeau, op.cit., p.344.

lii Ibid., p.399.

liii Ibid., p.527.

liv Ibid., p.529.

lv Ibid., p.293.

lvi Ibid., p.92.

lvii Ibid., p.94. 
lviii Ibid., p.239.

lix Idem.

${ }^{1 x}$ Ibid., p.589.

lxi Ibid., p.114.

lxii Ibid., p.115.

lxiii Ibid., p.147.

Ixiv Ibid., p.361.

lxv Ibid., p.454.

lxvi Ibid., p.456.

Ixvii Idem.

lxviii Idem.

lxix Ibid., p.116.

${ }_{1 x x} \mathrm{AFP}$ «Football, soupçons de terrorisme, investissements : quel avenir pour la relation France-

Qatar?» https://francais.rt.com/france/39272-football-soupcons-terrorisme-investissements-quelavenir-relation-france-qatar

lxxi Suaudeau, op.cit., p.20.

lxxii Ibid., p.22.

Ixxiii Ibid., p.24.

lxxiv Ibid., p.76.

${ }^{1 \times x v}$ AFP, op.cit., «Football, soupçons de terrorisme, investissements : quel avenir pour la relation France-Qatar?»

Ixxvi Suaudeau, op.cit., p.24.

lxxvii Ibid., p.530.

lxxviii Chloé Guille, «Lutte contre la radicalisation : marginalisation des musulmans »,

http://www.cipadh.org/fr/lutte-contre-la-radicalisation-marginalisation-des-musulmans

lxxix Suaudeau, op.cit., p.557.

Ixxx Ibid., p.118.

lxxxi Bertrand Badie Professeur des universités à l'Institut d'études politiques de Paris, directeur du cycle « Relations internationales », Yasmine Boudjenah Première adjointe (PCF) au maire de Bagneux (Hauts de- Seine), Paul Quilès Ancien ministre, animateur de Gauche Avenir, « Comment lutter efficacement contre le terrorisme ? », entretiens croisés réalisés par Dany Stive, 16 janvier 2015, https://humanite.fr/comment-lutter-efficacement-contre-le-terrorisme-562976

Ixxxii Suaudeau, op.cit., p.120.

lxxxiii Ibid., p.534.

Ixxiv Doudou Diène, Rapporteur spécial de l'ONU sur le racisme de 2002 à 2008, «Crise identitaire du monde occidental $»$, https://www.cairn.info/revue-internationale-et-strategique-2009-3-page-93.htm Ixxxv Idem.

lxxxvi Suaudeau, op.cit., p.592.

lxxxvii Institut malien de recherche action pour la paix (IMRAP), «Au-delà de l'idéologie et de l'appât du gain : trajectoires des jeunes vers les nouvelles formes de violence en Côte d'Ivoire et au Mali », $\mathrm{http}$ //bamada.net/terrorisme-et-violence-quest-ce-qui-pousse-les-jeunes-vers-les-groupes-extremistes lxxxviii Suaudeau, op.cit., p.202.

lxxxix Ibid, p.529.

${ }^{x c}$ Garello Jacques, économiste français, professeur émérite à l'Université Aix-Marseille III, président d'honneur de l'Association pour la liberté économique et le progrès social (ALEPS), « Face à la barbarie, toujours et encore l'humanisme $»$, https://www.contrepoints.org/2015/07/29/216023-face-ala-barbarie-toujours-et-encore-lhumanisme

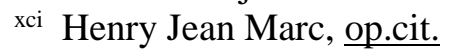




\section{Bibliographie}

\section{I-Corpus}

- SUAUDEAU Julien, Dawa, Editions Robert Laffont, 2014, Paris.

\section{II-Autres œuvres :}

- Camus Albert, L’Homme révolté, Gallimard, Paris,1951.

- Camus Albert, avec la contribution de Levi-Valensi Jacqueline, Garapon Antoine et Salas Denis, Réflexions sur le terrorisme, Editions Nicole Philippe, 2002, France.

- Cossery Albert, Euvres Complètes II, Editions Joëlle Losfeld, Paris, 2005.

- $\quad$ El Aswany Alaa, L’Immeuble Yacoubian, Actes Sud, 2006.

\section{III-Ouvrages de critique}

- Duchet Claude, Sociocritique, Editions Fernand Nathan, 1979, Paris.

- Goldmann Lucien, Pour une sociologie du roman, Editions Gallimard, 1964, Paris.

- Zima V. Pierre, Manuel de sociocritique, Editions Picard, 1985, Paris.

\section{Sitographie}

- Chloé Guille, «Lutte contre la radicalisation : marginalisation des musulmans », http://www.cipadh.org/fr/lutte-contre-la-radicalisation-marginalisation-des-musulmans

- Bertrand Badie Professeur des universités à l'Institut d'études politiques de Paris, directeur du cycle «Relations internationales », Yasmine Boudjenah Première adjointe (PCF) au maire de Bagneux (Hauts de- Seine), Paul Quilès Ancien ministre, animateur de Gauche Avenir, «Comment lutter efficacement contre le terrorisme ? », entretiens croisés réalisés par Dany Stive, 16 janvier 2015, https://humanite.fr/comment-lutterefficacement-contre-le-terrorisme-562976

- Bourhaban Samira, "Singularité et multiplicité des relations fraternelles : voyage en terre fraternelle $»$, in Cahiers critiques de thérapie familiale et de pratiques de réseaux, 2004/1 (no 32), Fratrie, à quel prix ? https:// www.cairn.info/revue-cahiers-critiques-detherapie-familiale-2004-page-23.htm

- Deffarges Thierry, Sur la nature et les causes du terrorisme, Une revue de la littérature économique, https://www.cairn.info/revue-tiers-monde-2003-2-page-369

- Doudou Diène, Rapporteur spécial de l'ONU sur le racisme de 2002 à 2008, «Crise identitaire $\mathrm{du}$ monde occidental », https://www.cairn.info/revue-internationale-etstrategique-2009-3-page-93.htm

- El Khadir Mahmoud, «Le terrorisme, les causes et les remèdes », Université Mohammed I Faculté de Droit - Licence 2005, https://www.memoireonline.com/01/07/330/m_le-terrorisme-les-causes-et-lesremedes.html

- «FLN et OAS : deux terrorismes en guerre d'Algérie », In Revue Européenne d'Histoire / European Review of History, vol. 14, $\mathrm{n}^{\circ} 3$, septembre 2007, p.325-342, https://halshs.archives-ouvertes.fr/file/index/docid/541818/.../FLN_et_OAS.doc 
- Galanopoulo Léa, «Le gène de la violence : un instrument politique », https://sciencescritiques.fr/le-gene-de-la-violence-un-instrument-politique/

- Garello Jacques, économiste français, professeur émérite à l'Université Aix-Marseille III, président d'honneur de l'Association pour la liberté économique et le progrès social (ALEPS), « Face à la barbarie, toujours et encore l'humanisme ", https://www.contrepoints.org/2015/07/29/216023-face-a-la-barbarie-toujours-etencore-lhumanisme

- Institut malien de recherche action pour la paix (IMRAP), «Au-delà de l'idéologie et de l'appât du gain : trajectoires des jeunes vers les nouvelles formes de violence en Côte d'Ivoire et au Mali », http://bamada.net/terrorisme-et-violence-quest-ce-qui-pousse-lesjeunes-vers-les-groupes-extremistes

- Leroux Georges, Hazam Olga, «Islam, terrorisme, un amalgame inquiétant », http://www.spiralemagazine.com/dossier-magazine/islam-islamisme-terrorisme-unamalgame-inquietant

- Lépine Élise, « Dawa, roman d'un Français en colère, Quand la fiction puise dans la réalité sociale, la littérature fait l'effet d'un coup de poing. La preuve avec le livre de Julien Suaudeau », http://www.lepoint.fr/culture/dawa-roman-d-un-francais-en-colere30-03-2014-1807110_3.php

- Mamdani Mahmood, «La CIA et la fabrique du terrorisme islamiste », https://www.ababord.org/La-CIA-et-la-fabrique-du-terrorisme-islamiste

- Robert Claude, « Terrorisme islamiste: quelles sont les vraies causes ? ", https://www.contrepoints.org/2017/01/02/261277-vraies-causes-du-terrorismeislamiste

- Rouibah Hicham, doctorant en socio-économie et en anthropologie politique à l'EHESS (Ecole des hautes études en sciences sociales) et à l'IRD (Institut de recherche et développement), «Islamiste / islamique, quelle différence et quel lien avec le terrorisme »», https://www.saphirnews.com/Islamiste-islamique-quelle-difference-etquel-lien-avec-le-terrorisme_a20584.html)

- Spiteri Pierre, Professeur Emérite des Universités, «La stratégie terroriste menée par le F.L.N : une stratégie hélas, efficace, », http://www.clan-r.org/portail/IMG/pdf/Lastrategie-terroriste-menee-par-le-FLN-une-strategie-helas-efficace.pdf

- Taubes Isabelle, « I'm just a mégalo ", www.psychologie.com/Moi/seconnaitre/comportement/articles-dossiers/l-m-just-a-megalo

- Telhard de Chardin Pierre, Paléontologue et philosophe, «L'amour est la plus universelle, la plus formidable et la plus mystérieuse des énergies cosmiques » in « Qu'est-ce que l'amour ? Valeur, énergie ou sentiment ? ", http://www.etreproactif.com/quest-ce-que-lamour-valeur-energie-ou-sentiment-etreou-faire/

- Tursz Anne, « Pourquoi et comment devient-on terroriste ? », http://www.huffingtonpost.fr/anne-tursz/trajectoire-terroristes-france_b_7068514.html

- 6Medias, « Terrorisme : quel est le profil des djihadistes ? ", http://www.lepoint.fr/terrorisme-quel-est-le-profil-des-djihadistes-php 19-08-2017215001_23) 